\title{
BMJ Open Quest for certainty regarding early discharge in paediatric low-risk febrile neutropenia: a multicentre qualitative focus group discussion study involving patients, parents and healthcare professionals in the UK
}

\author{
Jessica E Morgan, ${ }^{1}$ Bob Phillips, ${ }^{1,2}$ Lesley A Stewart, ${ }^{1}$ Karl Atkin ${ }^{3}$
}

To cite: Morgan JE, Phillips $B$, Stewart LA, et al. Quest for certainty regarding early discharge in paediatric lowrisk febrile neutropenia: a multicentre qualitative focus group discussion study involving patients, parents and healthcare professionals in the UK. BMJ Open 2018;8:e020324. doi:10.1136/ bmjopen-2017-020324

- Prepublication history and additional material for this paper are available online. To view these files, please visit the journal online (http://dx.doi org/10.1136/bmjopen-2017020324).

Received 30 0ctober 2017 Revised 2 February 2018 Accepted 6 March 2018

D) Check for updates

${ }^{1}$ Centre for Reviews and Dissemination, University of York, York, UK

${ }^{2}$ Department of Paediatric Haematology and Oncology, Leeds Teaching Hospitals NHS Trust, Leeds, UK

${ }^{3}$ Department of Health Sciences, University of York, York, UK

Correspondence to

Dr Jessica E Morgan;

jess.morgan@york.ac.uk

\section{ABSTRACT}

Objectives A systematic review of paediatric low-risk febrile neutropenia found that outpatient care is safe, with low rates of treatment failure. However, this review, and a subsequent meta-ethnography, suggested that early discharge of these patients may not be acceptable to key stakeholders. This study aimed to explore experiences and perceptions of patients, parents and healthcare professionals involved in paediatric febrile neutropenia care in the UK.

Setting Three different centres within the UK, purposively selected from a national survey on the basis of differences in their service structure and febrile neutropenia management.

Participants Thirty-two participants were included in eight focus group discussions.

Primary outcomes Experiences and perceptions of paediatric febrile neutropenia care, including possible future reductions in therapy.

Results Participants described a quest for certainty, in which they attempted to balance the uncertainty involved in understanding, expressing and negotiating risk with the illusion of certainty provided by strict protocols. Participants assessed risk using both formal and informal stratification tools, overlaid with emotional reactions to risk and experiences of risk within other situations. The benefits of certainty provided by protocols were counterbalanced by frustration at their strict constraints. The perceived benefits and harms of previous inpatient care informed participants' appraisals of future treatment strategies.

Conclusions This study highlighted the previously underestimated harms of admission for febrile neutropenia and the paternalistic nature of decision making, along with the frustrations and challenges for all parties involved in febrile neutropenia care. It demonstrates how the same statistics, generated by systematic reviews, can be used by key stakeholders to interpret risk differently, and how families in particular can view the harms of therapeutic options as different from the outcomes used within the literature. It justifies a reassessment of current treatment strategies for these children and further exploration of the potential to introduce shared decision making.
Strengths and limitations of this study

- This study involves new voices in the discussion of febrile neutropenia management, particularly healthcare professionals.

- It specifically explored disease-specific factors involved in decision making about early discharge.

- The inclusion of multiple centres allows for an understanding of service design and centre culture on participants' perceptions.

- Challenges related to the groups which proved difficult to recruit, including young people and those who have a limited ability to communicate in English.

- The small size of some of the focus groups may be considered a limitation by some. The implications of this are discussed further in the text.

\section{BACKGROUND}

Febrile neutropenia is the most common life-threatening complication of treatment for childhood cancer and carries a risk of sepsis, including intensive care admission and death. ${ }^{1}$ However, over $50 \%$ of children have no significant sequelae or clinically or microbiologically defined infection. ${ }^{2} 3$ Risk stratification tools may help distinguish those with high-risk febrile neutropenia from those with lower risk of significant complications, though it should be recognised that these tools have some challenges, and a universally agreed 'gold standard' tool has yet to be defined. ${ }^{3}$ Current treatment for children with febrile neutropenia in the UK consists of admission to hospital for at least 48 hours, with administration of empirical intravenous antibiotics. $^{4}$

It has been suggested that reducing therapy for children with low-risk febrile neutropenia could improve quality of life, reduce 
hospital acquired infections and reduce costs to healthcare. $^{5-8}$ Our recent systematic review found that early discharge did not increase intensive care admissions or death, but did appear to increase the risk of readmission to hospital, from around $2.2 \%$ to $14 \% .{ }^{9}$ Furthermore, there was a suggestion that reduced therapy options may not be acceptable to families and professionals as consent rates to the included trials were relatively low. Few other studies have explored family and professional preferences for location of therapy. In one study, interviews using a threshold technique found that just $53 \%$ of parents and $71 \%$ of professionals would choose outpatient treatment for low-risk paediatric febrile neutropenia. ${ }^{10}$ A meta-ethnography of the existing qualitative literature surrounding early discharge revealed that there may be challenges surrounding practical logistics, and social or emotional issues, influenced by fear, timing and resources. ${ }^{11}$ However, the data focused almost exclusively on parental views, lacked exploration of the influence of healthcare settings and had little disease-specific data about febrile neutropenia.

This study spoke to patients, parents and healthcare professionals involved in paediatric low-risk febrile neutropenia, to build understanding about perceptions of early discharge and so inform future policy and practice. We aimed to explore the contextual features that might influence the experience of individual patients and families, including previous experiences of febrile neutropenia, family structure and background and healthcare service design and culture. This paper presents one of the key themes from the findings, with the remainder presented elsewhere, to enable richer explorations within the limits of article word counts.

\section{METHODS}

National Health Service Research Ethics Committee (ref 15/YH0208) and study sites' Research and Development approval was obtained prior to commencement of the research.

\section{Study site identification and recruitment}

Data from a recent UK survey were used to identify centres with different approaches to risk stratification, low-risk protocols, shared care services and geographical spread of patients. ${ }^{12}$ Three centres were purposively selected to enable investigation of the role of various centre-level factors and then approached through professional groups within the Children's Cancer and Leukaemia Group.

Centres 1 and 2 both have around 25 inpatient beds at the primary treatment centres (PTCs) and see 100-150 new cases of childhood cancer per year. Centre 1 has minimal care of febrile neutropenia in its shared care centres. Centre 2 has some shared care services, but the majority of cases of febrile neutropenia are managed in the PTC. Centre 3 treats over 160 new children per year and has more than 30 inpatient beds. They have a strong shared care network, and most low-risk febrile neutropenia is managed within Paediatric Oncology Shared Care Units (POSCUs).

Centres 1 and 3 closely followed the current National Institute for Health and Care Excellence (NICE) guidelines for febrile neutropenia. ${ }^{13}$ Centre 2 used a higher threshold to define fever and did not use a risk stratification tool, although they were reviewing their policy. All centres' protocols involved at least 48 hours admission with intravenous antibiotics for at least 24 hours.

The study opened to recruitment in July 2015, and the final focus group discussion took place in March 2016.

\section{Identification, recruitment and consent of participants}

Four focus group discussions were planned in each study site, for each of: patients (aged 13-18 years), parents of teenagers (13-18 years), parents of younger children (under 13 years) and healthcare professionals (doctors and nurses working in paediatric haematology and oncology services). Families were invited if they were at risk of low-risk febrile neutropenia or within 6 months of being at risk, assessed by the modified Alexander rule. ${ }^{14}$ This rule was chosen as it is the risk stratification tool advised by the UK's NICE guidelines. ${ }^{13}$ Patients receiving palliative care alone were excluded. Parent-child dyads and parent couples were not explicitly recruited, but could be included.

Families were identified and invited to participate by their local team, then contacted by JEM to establish a relationship and answer further research questions. Healthcare professionals were invited through local team meetings and emails sent by the site-specific collaborator. Written consent was obtained. Participants had the right to withdraw at any point up to 2 weeks following the focus group discussion. Participants received travel expenses and a £20 Amazon voucher for their attendance at the group. This reflects INVOLVE guidance for participant remuneration. ${ }^{15}$

\section{Focus group discussions}

Each group had between 3 and 7 participants and lasted between 45 and $86 \mathrm{~min}$ (median $73 \mathrm{~min}$ ). Focus groups were performed at a site suitable for participants, for most this was within the hospital building, but other facilities such as local library meeting rooms were also used. JEM, a Clinical Research Fellow, moderated all groups, with an assistant present. This study forms part of her $\mathrm{PhD}$, and she has completed Level 7 training in focus group moderation and qualitative methodology. Participants were aware of the study's aims and objectives and of JEM's research background. If directly asked, she confirmed her medical professional background. Focus group topic guides are included in online supplementary resource 1. Encrypted digital audio recordings of each discussion were obtained, then transcribed and anonymised by JEM. A research journal was kept throughout. 


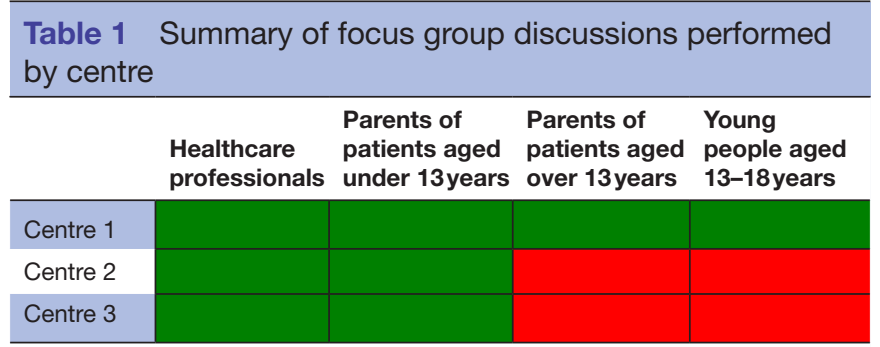

Green-focus group performed, red-focus group desired but precluded by poor recruitment.

\section{Analysis}

This study used a constant comparison approach. Each transcript was individually coded by JEM with input from KA. No analytical software was used. At the intragroup comparison stage, individual voices were followed through the focus groups to identify codes which occurred more frequently, or with different quality, dependent on the characteristics of individuals. Discussions were then compared with other groups of participants (healthcare professionals, parents of under 13 years, parents of teenagers and young people) and other centres. At this intergroup stage, the triangulation of centres and participant groups was explicitly explored. The two stages were then overlaid to provide a network through which the general iterative themes were compared and mapped prior to representing the final framework. Following presentations of the findings, participants have confirmed that they agree with the analytical findings.

\section{RESULTS}

\section{Participants}

Thirty-two participants were included in eight focus group discussions. Four additional focus group discussions were intended but were precluded by poor recruitment of young people and parents of teenagers in centres 2 and 3. Table 1 provides a summary of the focus groups performed at each centre, and table 2 provides a more detailed summary of the focus group compositions.

\section{Understanding, expressing and negotiating risk}

In all centres, healthcare professionals struggled to cognitively separate different febrile neutropenia risk groups, tending to think about children as having 'febrile neutropenia', rather than low-risk or high-risk episodes.

4: I think it's just I've been here for an awful long time and that's what we've done and I know it's the small number of children that you can remember that just... that just do... collapse within less than 8 hours...

Mod: without giving us patient identifiable data, could you describe one?

4: mm... oh God... erm... ermm... yeah probably the most vivid one was when I was over at [another hospital] ... so I couldn't even tell you the name of the patient... erm... but walked onto the ward having had a single fever at home... and within... within minutes we were resuscitating him...

1: but he wouldn't be low risk would he?

4: he wouldn't no but that's the bit that I don't know why but that's the bit that sticks in my mind... (Centre 1 , nurse (4) and doctor (1))

It is not surprising that the healthcare professional participants had emotional responses to the idea of early discharge; generally anxiety or fear. One participant physically shivered at the mention of outpatient treatment:

1: it's not like they won't be febrile at home... they'd be going home febrile wouldn't they (murmur of agreement) so then you'd have to work out what...

4: [4] shivered again!!! (laughter) (Centre 1, doctor

(1) and nurse (4))

Parents and young people group participants were unfamiliar with formal risk stratification. Instead, parents employed their own methods to establish the dangers posed by an episode of febrile neutropenia. Participants differentiated episodes into those where the child appeared unwell, was not their usual self and caused the parents to worry from those in which the child had a fever but their behaviour did not otherwise concern their parents. As such, the idea of being 'well' or 'unwell' formed an instinctive risk stratification 'tool'. Families envisaged that the management of these groups would be adapted according to the severity of 'unwellness'.

When formal risk stratification tools were introduced into the parents' focus group discussions, participants spoke about how clearly knowing whether their child was at low or high risk of significant complications facilitated their own decision making about preferred care.

2: ...now that I know that you've said that he's in a lower risk group then actually maybe I wouldn't have panicked quite so much and thought you know... and if the option would have been there... I would have probably gone with it but not knowing that information and... and just being told 38 degrees he's got to go in and.... And I just follow protocol... I follow the rules... (Centre 1, parents of under 13s)

This acceptance by parents of risk stratification as a concept and their current use of a similar assessment strategy suggests that increased communication of the level of a child's risk may support shared decision making between families and healthcare professionals. Furthermore, explicitly stating the level of risk for a child may quieten the emotional responses that healthcare professionals have towards reduced therapy regimens.

The challenge of deciphering statistical evidence regarding risks was evident, even within the healthcare professional groups. In one group, misunderstandings about systematic review methodology and confusion over statistical issues, such as power, led to mistrust of the evidence. 
Table 2 Table of focus group composition

\begin{tabular}{|c|c|c|c|c|c|c|}
\hline Location & Group & $\begin{array}{l}\text { Number of } \\
\text { participants }\end{array}$ & M:F ratio & Age & $\begin{array}{l}\text { Ethnicity (self- } \\
\text { defined) }\end{array}$ & Composition notes \\
\hline Centre 1 & Young people & 3 & $0: 3$ & $\begin{array}{l}15- \\
16 \text { years }\end{array}$ & 3 White British & $\begin{array}{l}\text { Two participants with Hodgkin's disease, one with ALL. One } \\
\text { participant with no febrile neutropenia episodes, one with } \\
\text { one low-risk episode, one with six high-risk episodes. All } \\
\text { episodes treated at PTC (two episodes started at POSCU } \\
\text { but transferred to PTC). No ICU admissions. One course } \\
\text { of chemo delayed following high-risk episode. Admissions } \\
2-10 \text { days (most 5-6 days). }\end{array}$ \\
\hline
\end{tabular}

\begin{tabular}{|c|c|c|c|c|c|c|}
\hline Centre 1 & $\begin{array}{l}\text { Parents of over } \\
13 \text { years }\end{array}$ & 5 & $2: 3$ & $\begin{array}{l}41- \\
53 \text { years }\end{array}$ & All White British & $\begin{array}{l}\text { Three nuclear families with } 2-3 \text { children. One blended family } \\
\text { (both parents present), with three children. One degree- } \\
\text { level education, one A level, one ONC, two GCSE level. } \\
\text { All employed except one who is semiretired (to care for } \\
\text { child). Partners same educational and employment levels. } \\
\text { All own house, four with mortgage. Four have children who } \\
\text { participated in young people's group. } \\
\text { Affected children 13-16years old. Two with Hodgkin's } \\
\text { disease, one with ALL, one with relapsed ALL. One patient } \\
\text { with no febrile neutropenia episodes, one with one low- } \\
\text { risk episode, one with six high-risk episodes, one with } \\
\text { seven high-risk episodes and one low-risk episode. All } \\
\text { episodes treated at PTC (two episodes started at POSCU } \\
\text { but transferred to PTC). No ICU admissions. Two courses } \\
\text { of chemo delayed following high-risk episodes. Admissions } \\
2-10 \text { days (most 5-6 days). }\end{array}$ \\
\hline Centre 1 & $\begin{array}{l}\text { Healthcare } \\
\text { professionals }\end{array}$ & 7 & $3: 4$ & $\begin{array}{l}30- \\
51 \text { years }\end{array}$ & $\begin{array}{l}6 \text { White British, } 1 \\
\text { Chinese }\end{array}$ & $\begin{array}{l}\text { Three medical (SHO, registrar and consultant), four nursing } \\
\text { (bands 5-7). 1-13years at current grade. 1-24 years at current } \\
\text { centre. }\end{array}$ \\
\hline Centre 2 & $\begin{array}{l}\text { Healthcare } \\
\text { Professionals }\end{array}$ & 3 & $0: 3$ & $\begin{array}{l}43- \\
60 \text { years }\end{array}$ & $\begin{array}{l}1 \text { White British, } 1 \\
\text { Indian (British), } 1 \\
\text { Pakistani }\end{array}$ & $\begin{array}{l}\text { Two medical (registrar, consultant), one nursing. } \\
7 \text { months- } 20 \text { years at current grade. } 7 \text { months }-28 \text { years at } \\
\text { current centre. }\end{array}$ \\
\hline Centre 3 & $\begin{array}{l}\text { Healthcare } \\
\text { Professionals }\end{array}$ & 3 & $0: 3$ & $\begin{array}{l}30- \\
55 \text { years }\end{array}$ & All White British & $\begin{array}{l}\text { One medical, two nursing (band } 7) .3-12 \text { years at current } \\
\text { grade. } 5-12 \text { years at current centre. }\end{array}$ \\
\hline
\end{tabular}

ALL, Acute Lymphoblastic Leukaemia; GCSE, General Certificate of Secondary Education; HNC, Higher National Certificate; ICU, intensive care unit; ONC, Ordinary National Certificate; PICU, paediatric intensive care unit; POSCU, Paediatric Oncology Shared Care Unit; PTC, primary treatment centre; SHO, Senior House Officer. 
"Mod: ...2660 episodes of data, how much more would people need to be more confident in this number (points to treatment failure rate)?

[ongoing discussion]

1 : is that international?

Mod: yes it's international around the world

2: so if it's all round the world?

[additional discussion]

2: so actually you could say it's a small number if it's...

1: there's the whole world...

2: yeah couldn't you? and how many within each study? That would be the other thing

[further discussion]

1: because if the numbers were high, people would be more convinced that this is real, I think if er... you know I'd see, for instance this is just an example, if you had 500000 episodes and then you saw this, you'd say yeah that's it, this is what we need to go for (murmur of agreement from (2)) but two and a half thousand odd is not going to be convincing. (Centre 2, doctor (1) and nurse (2))

Alongside these statistical misunderstandings, in all healthcare professional groups, there were moments within the discussion where comments made were inconsistent with other beliefs they held. In centre 2 , one participant stated "but if you look at the $0.1 \%$ risk [of PICU admission or death in low risk febrile neutropenia], it's still high, in that group, because your range is between $0.03 \%$ to $0.3 \%$, and you've got $0.1 \%$ ". Considering a $0.1 \%$ risk of paediatric intensive care unit admission or death to be high seems unusual: in a field where $3 \%$ of children with cancer die of infection and around $15 \%$ die of progressive disease. ${ }^{116}$

One method that healthcare professional participants used to help understand the risk statistics and express the inconsistency of their discussions was to compare the risks in febrile neutropenia to other clinical situations, both within the context of other haematology and oncology conditions and within other specialties, particularly general paediatrics.

Discussion by professionals about their own decision making revealed and acknowledged tension between making judgements based on research evidence and individual emotional experience.

2: I think the interesting thing there for me [Mod] is that you've presented us with the evidence (laughter from others) which is by far and away saying that this is a safe thing to do...

4: we've chosen to ignore it...

2: but we've chosen to ignore it... so we're practicing non-evidence based anecdotal medicine (ongoing laughter) but it's what we're comfortable with...
Mod: ok... so what factors played the role in making that decision...

2: non evidence based anecdotal...

4: anxiety...

(laughter and indiscernible mutterings) (Centre 1, doctor (2) and nurse (4))

In centre 3, professionals used fewer anecdotal accounts of patient deteriorations and appeared to have a less emotional, and more positive, response to early discharge.

Professional participants were very clear about the extent of influence that healthcare staff should have over families' perceptions of risk. In the following quote, a participant outlines the dilemma of how to communicate about risk.

....and then you have to try and put the frighteners on them and you have to gauge that right as to how... because I've had people saying oh I... oh I know... we've got four hours to wait... and I say well sometimes children deteriorate more rapidly than that.... erm... but it's really difficult to know quite how... how scary to be with them isn't it? (Centre 3, doctor)

Throughout this theme, it is clear that participants struggled with the uncertainty of risk. They sought the illusion of certainty, aiming to find security in the absolute, irrespective of the reality of its non-existence. This may be founded in limited understanding or familiarity with statistical concepts. However, the assessment of risk is not a purely technical act but instead a political and social construct, with many underlying social, emotional and cultural influences.

\section{Articulating and interpreting protocols}

All the documents discussed in this manuscript are entitled guidelines, suggesting flexibility in the use of their recommendations. However, participants used the word protocol almost exclusively and appeared to understand and use these documents as formal and rigid policies. We have used their terminology.

Although the direction given by protocols provided reassurance to healthcare professionals, protocols were interpreted as controlling and limited decisions by implying an inability to deviate from them, resulting in frustration:

1: I think the other thing that makes it frustrating its very protocol driven innit?

(2: (at the same time) yeah)

1: I think people stick rigidly to 48 hours as though it's a magic number and nobody can go home at 43 hours and etcetera... so I think it's very very rigid err which is done for good reasons but I think if you dare to suggest that you veer from that you're scorned upon... (Centre 1, doctors)

Professionals described risks of working outside the protocol, in particularly relating to receiving criticism from colleagues, with groups only briefly referring to 
the safety risks to patients of deviating from a protocol. Notably, they referred to the risks to patients of other professionals departing from the protocol, but the risks of criticism refer to their own practice.

Participants varied in how much flexibility they thought there should be within a protocol. Centre 1 spoke of a strict protocol with minimal deviations and were willing to accept a greater degree of frustration because of this. Centre 2 gave considerable weight to clinical reviews, with minimal references to protocols as a guiding feature. Combining these approaches, professionals at centre 3 spoke about the integration of protocol and clinical judgement:

1: like you've got the protocol there but actually if you know your patient... me and the consultant might make a decision on our patient that isn't what the protocol says but we're happy with that clinical decision (Centre 3, nurse)

Parents' main concern regarding protocols related to the fact that discharge rarely occurred at the point where it was theoretically possible in the protocol. Parents distinguished between appropriate delays due to the child's ill health and those which they felt were in some way avoidable. They voiced their frustration about how services, and decision making, were conditional on the timing of a child's presentation with low-risk febrile neutropenia. The staff working out of hours may not be as senior or as experienced as those who provide routine services, and as such, parents recognised that they may make different judgements about the levels of risk involved or may not feel able to take on the responsibility of discharging a child.

Astutely, parents in a number of groups identified that blood culture results were a significant factor in professionals' decision making in regard to discharge, and that most protocols demanded a negative blood culture result before a child could be evaluated for discharge. Delays in processing could result in substantial delays in decision making.

1: then my frustration comes with the process and blood culturing and that, towards the end of a stay, is what just really really narks me, that we actually spend at least an extra day in because they don't culture the bloods when you get in straight away, so if we were admitted on Monday at noon or after, they don't start culturing the bloods until Tuesday at 9am... (Centre 3 , parent of under $13 \mathrm{~s}$ )

Thus, blood cultures symbolise the potential for parents to 'escape' from hospital. Delays in obtaining results capture parents' greatest dislike of current services and protocols; unnecessarily prolonged hospital stays with an apparently well child.

\section{Preferences for care}

Participants' past experiences help to inform and find balance between the uncertainty of risks and the certainty of protocols. The findings outlined within this section all impacted on how participants formed their ideas regarding early discharge. Participants discussed many issues similar to those found in the earlier meta-ethnography. ${ }^{11}$ Participants spoke of the tiring nature of travelling, particularly at night time or during the rush hour. Distance from the hospital played a key role in whether early discharge regimens would be accepted, with families living close to the treating hospital more likely to accept outpatient care. Participants in centre 3, barely mentioned travelling, presumably because they already live close to their treating POSCUs.

Parents discussed finances, related to travel, parking, hospital food and lost income. This was vividly described by some participants, including the loss of a self-run business, repossession of a car and the receipt of benefits such as free school meals. The unpredictable nature of febrile neutropenia episodes proved more problematic than scheduled attendances for review or chemotherapy as it did not allow them to plan other aspects of their lives, chiefly their work.

Participants spoke about the psychological impact of admissions on their child who would become quiet, anxious or angry when their temperatures were checked at home, and they anticipated that they might need to travel to hospital. Following discharge, psychological effects would continue for some time:

1: we're definitely a more stressed family when he's admitted... definitely... and it takes a long time for the family to get back on track, it's not just he's home and we're all fine, you actually have to completely collapse and rebuild and that takes a couple of days... (centre 3, parents of under 13s)

Parents described split families, where one parent was in hospital with the affected child and the other at home with their siblings, with occasional 'handovers' of care, which impacted on their relationships as couples. The parent who took on the role of primary carer during admissions often had a deeper relationship with the child when they returned home as well, and the other parent could feel rejected or 'worthless'. They expressed guilt over their choices when faced with the differing needs of their children.

Professionals mostly spoke about the experiences of families, with very few references to their own perspectives, even when directly asked. They mentioned each of the key issues mentioned above but provided less detail and did not identify more nuanced issues about the patient experience. This situation resulted in double silencing of both parents and professional voices.

Six newly identified difficulties were discussed by participants in this study. First, all participants spoke clearly about how these are generally well children who are active, noisy and boisterous on a ward. They reported boredom and frustration during admissions and were concerned about their child disturbing other children who were more unwell. Second, parents worried about 
'hospital acquired infection' and spoke about the risks of wards or hospitals which were not clean. Third, participants spoke about the impact of 'source isolation' in two ways. First, well children who are source isolated are usually even more bored or frustrated. Second, where source isolation beds are limited, participants knew that these are unavailable to sicker patients when children with low-risk febrile neutropenia are admitted. Parents expressed guilt for using these resources. Staff expanded on the issue of bed pressure (the fourth new difficulty), describing intense challenges faced by health services, where numbers of bed spaces are reduced and costs of bed occupancy are high.

The fifth challenge, discussed only by parents and young people, related to side effects of treatments, particularly diarrhoea caused by antibiotics, necessitating source isolation, and might also cause the child to need intravenous fluids or total parenteral nutrition, delaying their discharge. Parents voiced a clear preference for reduced amounts of antibiotics, particularly those given intravenously. In addition, parents spoke about concerns regarding antibiotic resistance and how this might impact on their child in the future. These issues were not raised in the healthcare professional discussions.

Sixth, healthcare professionals in centre 3 discussed the long-term psychological impact of current febrile neutropenic care.

....as a late effects nurse... the risk of repeated hospitalisations and family seeing their child as sick and continuing to do so after the... and never really recovering from that sick child mentality... I don't know if that's a bit strong... I see the late effect of that when you've still got families who haven't been able to stop treating their child as a sick child right up until the child being in their early adulthood... I don't know how you'd ever measure that to balance it against that $0.1 \%$ risk... (Centre 3 , nurse)

Three benefits of inpatient care were described. First, was the idea that hospital is a safe place, where professionals looked after their child's health, though focused mainly on physical health. Parents stated that hospital was the place they would prefer their child to be if they judged them unwell. Families felt comforted by the presence of healthcare staff with whom they had good relationships and by the ritual of performing regular observations, although some groups did highlight that many observations could be done at home. Second, hospital can be fun. Young people and their parents enjoyed the input of youth support co-ordinators and activities that are organised in the hospital. This did not seem to play a major role in decision making, and parents were clear that they would still prefer a reduced therapy strategy. Third is the relational benefits that children and young people gained from the undivided attention of a parent, who, at home, might be distracted by other siblings and by the 'hubbub' of daily life.
When participants discussed the preferences of other participant groups, they often misunderstood perceived desires of others. For example, the young people anticipated that their parents would all rather receive inpatient care. This was at odds to the parents' stated preferences

1: if my mum agreed with the doctors, then I'd just do what she said, cos they don't like... when I'm saying something its normally cos it's what I'd prefer, when my mum tells me to do something its what's best for me... so... I'd probably whinge about having to staying in hospital but then... I'd just stay anyway.

2: I don't know... I don't think I'd really... I think my mum would just do what's right for me. Yeah (Centre 1 , Young people)

\section{DISCUSSION}

In summary, participants described a quest for certainty, in which they attempted to balance the uncertainty involved in understanding, expressing and negotiating risk with the illusion of certainty provided by strict protocols. Risks were assessed using both formal and informal stratification tools, overlaid with the emotional reactions to risk and experiences of risk within other situations. Professionals in particular demonstrated more emotional responses than might have been anticipated, which appeared most associated with centre culture rather than age or experience. Understanding statistical expressions of risk proved challenging for patients, parents and healthcare professionals. Meanwhile, the benefits of certainty provided by protocols resulted in frustration at the strict constraints they mandated. The perceived benefits and harms of inpatient care that participants had previously experienced informed their appraisals of future treatment strategies and provided them with both more confidence in their risk assessments and a greater desire for flexibility within protocols.

Throughout this study, the differences in focus between families and healthcare professionals became apparent, particularly concerning health. Professionals had a limited focus on physical health, almost entirely on the prevention of an intensive care admission or death. Their focus on individual children was relatively narrow, though they had a broader focus on the number of families impacted, taking into account the variety within their service population. Parents meanwhile focused on the broader aspects of child health, including side effects of interventions, social and emotional impacts and wider family health. However, they mostly concentrated on their individual child. Though they did discuss how others might differ in their opinions and desires when considering future services, it was the optimum regimen for their child and family that was put forward most strongly. These differences in objectives for future care are understandable given the responsibilities of families and healthcare professionals. However, stakeholders did not always understand each other's priorities. 
Linked to this difference in focus on health, participants discussed the most distressing aspects of being involved with children with febrile neutropenia differently according to their participant group. Healthcare professionals almost always discussed intensive care admission or death as their primary adverse experiences. Meanwhile, parents focused more on their experiences of poor care, such as delayed identification of positive culture growth, difficult venous access and the failure of professionals to identify what they perceived to be an unwell child. This highlights the differences in consideration of health between the two groups, while emphasising that many parents have no experience of death or intensive care admissions related to febrile neutropenia. Thus, their perceptions of risk of these events were somewhat different to those of professionals who often have experience of high-risk febrile neutropenia and thus develop strongly emotive reactions to suggestions of reductions in therapy. To families who experience more of the burdens of inpatient febrile neutropenia care, it is the failures to provide care focused on a holistic definition of health which play a more significant role in their experience.

These differences in focus further inform the interpretation of our previous systematic review and of healthcare research in general. ${ }^{9}$ The review used outcomes, collected by the primary studies, that reflect the focus of healthcare professionals-intensive care, death and healthcare service usage, as opposed to those of the families receiving care. We now see that when primary research is designed by professionals to detect these outcomes and fails to take into account the experiences of care that families value, these outcomes are also hidden in secondary research. Future research design should involve more public and patient involvement in the outcome setting stage, and the interpretation of quantitative research should involve discussions with patients and families so as to gain more insight into the different viewpoints on specific statistical findings, dependent on prior experiences and consideration of other benefits and harms.

Historically, determinism has shaped healthcare culture. ${ }^{17}$ In the deterministic worldview, predominant in early medicine, the role of physicians was to establish the definitive causes and treatments for disease. The clinician would strive to control each aspect of a patient's health so as to have control over their outcomes. ${ }^{17}$ Determinism inspires and promotes the creation of certainty, and thus where this cannot be achieved, the illusion of certainty can be considered a reasonable replacement as it provides a sense of control and reassurance within difficult situations. Understanding statistical risks and making decisions based on them is not prioritised, instead: "The goal is certainty, rather than learning how to live with uncertainty". ${ }^{17}$

In contemporary healthcare, there is no certainty. Shared decision making has been suggested as an appropriate step in overcoming determinism, recognising differences in focus and beginning to respect the rights of patients to have autonomy over their healthcare decisions. Shared decision making is most suited to situations in which evidence shows little difference in outcomes between treatment options, and thus patients' values or preferences might play a more important role in resolving this equipoise. ${ }^{18-21}$ In low-risk paediatric febrile neutropenia, evidence suggests that serious safety events are not affected by the location of care, and, as such, individualised judgements about other risks of readmission and patient preferences become more prominent. ${ }^{9}$ Specifically, within the literature, there has been a drive to use shared decision making "where the balance of risks and benefits varies widely in different medical, social and health care situations". ${ }^{18}$ Given the findings reported here, it might be considered that patients' social circumstances, along with the individual psychological impact of admission, might lead to a different balance of risks and benefits for each family affected by paediatric low-risk febrile neutropenia.

Practically, one approach which may be particularly useful in stimulating shared decision making conversations is to routinely risk stratify all patients and communicate this risk to families, following education regarding the differences in risk groups and in the management of febrile neutropenia. This will be further facilitated by ongoing work by the Predicting Infectious Complications of Neutropenic sepsis In Children with Cancer collaboration into the development of clear and robust risk stratification rules, so that there is universal agreement on the definition of children at low risk of septic complications.

\section{Strengths and weaknesses}

This study involves new voices in the discussion of febrile neutropenia management, particularly healthcare professionals and deepened consideration of the disease-specific factors which influence stakeholders' experiences and inform their decisions about future care. The inclusion of multiple centres allows for an understanding of the impact of service design and centre culture on participants' perceptions.

The majority of the challenges relate to recruitment difficulties, particularly of young people and those who have a limited ability to communicate in English. This reflects broader problems within the research community of engaging with participants from social disadvantaged groups and those from multicultural contexts. ${ }^{22}{ }^{23}$ Introducing Amazon vouchers to compensate participants for their time resulted in improvements in recruitment of young people and thus have helped to reduce the shortcomings. Future studies may wish to integrate other methodologies, including interviews or online focus groups for those who do not wish to participate in face-to-face groups, as well as options to participate using different languages for those who are unable to confidently take part in English language focus groups.

The small size of some of the focus groups may be considered a limitation by some as they may lead to less extensive discussions, and shy or dominating participants may have a more acute effect on the group. However, 
smaller groups have been purported to be beneficial in discussions involving complex subjects, or in groups of experts, such as healthcare professionals, and thus we feel are not a substantial disadvantage in this setting. ${ }^{22425}$

\section{CONCLUSIONS}

This study highlights the previously underestimated harms of admission for febrile neutropenia and the paternalistic nature of decision making, along with the frustrations and challenges for all parties involved in febrile neutropenia care. It justifies a reassessment of current treatment strategies for these children and further exploration of the potential to introduce shared decision making.

Acknowledgements Without doubt, the success of this study was made possible through the existence of a strong network of paediatric oncology and haematology services in the UK and more specifically through the availability of the regular febrile neutropenia audits performed by the Children's Cancer and Leukaemia Group. We thank all study participants for their honesty and engagement.

Contributors JEM designed the study, approached and liaised with centres, moderated focus groups, analysed the data and wrote the first draft of the manuscript. BP, LAS and KA provided substantial critical input into the design, performance and analysis of the study throughout the work and also critically revised the manuscript. All authors have given approval for the final version to be published.

Funding JEM was funded by Candlelighters Children's Cancer Charity for the duration of her PhD fellowship, of which this study forms a part.

Competing interests None declared.

Patient consent Obtained.

Ethics approval NHS Research Ethics Committee (ref 15/YH0208).

Provenance and peer review Not commissioned; externally peer reviewed.

Data sharing statement Given the nature of qualitative research, no additional unpublished data are available from this study.

Open Access This is an Open Access article distributed in accordance with the Creative Commons Attribution Non Commercial (CC BY-NC 4.0) license, which permits others to distribute, remix, adapt, build upon this work non-commercially, and license their derivative works on different terms, provided the original work is properly cited and the use is non-commercial. See: http://creativecommons.org/ licenses/by-nc/4.0/

(c) Article author(s) (or their employer(s) unless otherwise stated in the text of the article) 2018. All rights reserved. No commercial use is permitted unless otherwise expressly granted.

\section{REFERENCES}

1. Basu SK, Fernandez ID, Fisher SG, et al. Length of stay and mortality associated with febrile neutropenia among children with cancer. $J$ Clin Oncol 2005;23:7958-66.
2. Chisholm JC, Dommett R. The evolution towards ambulatory and day-case management of febrile neutropenia. Br J Haematol 2006;135:3-16.

3. Phillips RS, Lehrnbecher T, Alexander S, et al. Updated systematic review and meta-analysis of the performance of risk prediction rules in children and young people with febrile neutropenia. PLOS One 2012;7:e38300.

4. Johnson E, Phillips RS, Chisholm J, et al; CCLG National febrile neutropenia audit report 2015, 2015.

5. Ammann RA, Bodmer N, Hirt A, et al. Predicting adverse events in children with fever and chemotherapy-induced neutropenia: the prospective multicenter SPOG 2003 FN study. J Clin Oncol 2010;28:2008-14.

6. Sung L, Aplenc R, Alonzo TA, et al. Effectiveness of supportive care measures to reduce infections in pediatric AML: a report from the Children's Oncology Group. Blood 2013;121:3573-7.

7. Mustafa MM, Aquino VM, Pappo A, et al. A pilot study of outpatient management of febrile neutropenic children with cancer at low risk of bacteremia. J Pediatr 1996;128:847-9.

8. Teuffel O, Amir E, Alibhai SM, et al. Cost-effectiveness of outpatient management for febrile neutropenia in children with cancer. Pediatrics 2011;127:e279-e286.

9. Morgan JE, Cleminson J, Atkin K, et al. Systematic review of reduced therapy regimens for children with low risk febrile neutropenia. Support Care Cancer 2016;24:2651-60.

10. Sung L, Feldman BM, Schwamborn G, et al. Inpatient versus outpatient management of low-risk pediatric febrile neutropenia: measuring parents' and healthcare professionals' preferences. J Clin Oncol 2004;22:3922-9.

11. Morgan J, Cleminson J. Atkin K Qualitative synthesis of experiences of early discharge, with a focus on paediatric febrile neutropenia. Submiss.

12. Bate J, Gibson F, Selwood K, et al. A reaudit of current febrile neutropenia practice in UK paediatric oncology centres prior to implementation of NICE guidance. Arch Dis Child 2013;98:315-6.

13. NICE. NICE CG151 Neutropenic sepsis: full guideline. http://www. nice.org.uk/ (accessed 25 Sep 2013).

14. Dommett R, Geary J, Freeman S, et al. Successful introduction and audit of a step-down oral antibiotic strategy for low risk paediatric febrile neutropaenia in a UK, multicentre, shared care setting. Eur $J$ Cancer 2009;45:2843-9.

15. Mental Health Research Network and INVOLVE. Budgeting for involvement: practical advice for budgeting for actively involving the public in research studies, 2013.

16. National Cancer Intelligence Network. National registry of childhood tumours progress report. Oxford, UK: NRCT, 2012.

17. Gigerenzer G, Gaissmaier W, Kurz-Milcke E, et al. Helping Doctors and Patients Make Sense of Health Statistics. Psychol Sci Public Interest 2007;8:53-96.

18. Ferrer RL, Gill JM. Shared decision making, contextualized. Ann Fam Med 2013;11:303-5.

19. Berger Z. Navigating the unknown: shared decision-making in the face of uncertainty. J Gen Intern Med 2015;30:675-8.

20. Pollard S, Bansback N, Bryan S. Physician attitudes toward shared decision making: A systematic review. Patient Educ Couns 2015;98:1046-57.

21. Elwyn G. Idealistic, impractical, impossible? Shared decision making in the real world. Br J Gen Pract 2006;56:403-4.

22. Bloor M, Frankland J, Thomas M, et al; Focus groups in social research, 2001.

23. Barbour R. Doing focus groups. London: SAGE Publications, Ltd, 2007.

24. Morgan DL. Focus groups as qualitative research. 2nd ed. London: Sage Publications, Inc, 1997.

25. Barbour RS, Kitzinger J. Developing focus group research: Politics, theory and practice. London: SAGE Publications, Ltd, 1999. 